\title{
Inhaltsverzeichnis des 134. Bandes
}

\author{
Heft 1 und 2
}

Ausgegeben im Oktober 1971

H. Schulz (Zürich), W. Hoffmann (Münster) and G. M. Muchow (Toledo, Ohio), The average structure of $\mathrm{Mg}\left[\mathrm{Al}_{2} \mathrm{Si}_{3} \mathrm{O}_{10}\right]$, a stuffed derivative of the high-quartz structure. . . . . . . . . . . . . . . . .

Herbert Hauptman (Buffalo, New York), The probability distributions of several structure factors and their magnitudes. . . . . . . . . .

H. Brigitte Krause and Donald L. Gibbon (University Park, Pennsylvania), Ordering of $\mathrm{Mg}$ and $\mathrm{Nb}$ in the octahedral positions of the "cubic" perovskite structure of $\mathrm{Pb}_{3} \mathrm{MgNb}_{2} \mathrm{O}_{9}$. . . . . . . . . . . . . .

K. Anzenhofer and T.G. Hewitt (Amsterdam), The crystal and molecular structure of $\left[\mathrm{Ni}\left(\mathrm{C}_{5} \mathrm{H}_{8} \mathrm{O}_{2}\right)_{2}\left(\mathrm{H}_{2} \mathrm{O}\right)_{2}\right]\left(\mathrm{ClO}_{4}\right)_{2}$. . . . . . . . . .

Roland C. Rouse (Ann Arbor, Michigan), The crystal chemistry of diaboleite

Vasantha Pattabhi and K. Venkatesan (Madras), Automatic procedure for crystal-structure analysis with the heavy-atom technique. . . . .

Gulzari Lal, G. K. Chadha and G. C. Trigunayat (Delhi), Structure of an eighteen-layered polytýpe of cadmium iodide. . . . . . . . .

C. J. De Ranter and M. Van Dijck (Leuven), A one-term Gaussian representation of the x-ray scattering factor of core electrons . . . . . . . 97

Ekhard Salje (Hannover), Physikalische Eigenschaften von $\mathrm{KJO}_{3}$. . . . 107

George T. Faust and Waldemar T. Schaller (Washington, D.C.), Schoenfliesite, $\mathrm{MgSn}(\mathrm{OH})_{6}$. . . . . . . . . . . . . . . . 116

Kuniaki Kihara and Toshio Sudo (Tokyo), The structure of $\alpha$-type cesium antimony nonachloride, $\mathrm{Cs}_{3} \mathrm{Sb}_{2} \mathrm{Cl}_{9}$. . . . . . . . . . . . . 142

M. Korekawa und D. Philipp (München), Über zwei verschiedene Arten von Satellitenreflexen, beobachtet an einem Tieftemperatur-Plagioklas $\mathrm{An}_{16,5}$. . . . . . . . . . . . . . . . . . . . . 142

Surendra Nath Srivastava (Allahabad), Suggestion for improving Patterson and difference Fourier maps. . . . . . . . . . . . . . . . 148

\section{Vorläufige Mitteilungen-Preliminary notices}

Asok Banerjee (Calcutta), Crystal data of androsterone, $\mathrm{C}_{19} \mathrm{H}_{30} \mathrm{O}_{2}$. . . . . 152

Birendra Nath Das (Calcutta), Crystal data for testosterone, $\mathrm{C}_{19} \mathrm{H}_{28} \mathrm{O}_{2}$. . . 154

Kuniaki Kihara and Toshio Sudo (Tokyo), Two structure types of cesium antimony nonachloride, $\mathrm{Cs}_{3} \mathrm{Sb}_{2} \mathrm{Cl}_{9}$. . . . . . . . . . . 155

K. Huml and J.Jeiny (Prague), Crystal data for some $\beta$-diketone chelates of (1,6-dichloro-1,5-cyclooctadiene) rhodium . . . . . . . . 159 


\section{Heft 3 und 4}

Ausgegeben im November 1971

V. K. Agrawal (New Delhi) and G. K. Chadha and G. C. Trigunayat (Delhi), Crystal structures of two twelve-layered $\mathrm{CdI}_{2}$ polytypes . . . . . . 161

P. L. La Fleur (Nijmegen), Determination of dispersion and polarization of thermal plane waves in crystals . . . . . . . . . . . . . . . 166

P. Engel und W. Nowacki (Bern), Die Molekular- und Kristallstruktur von trans- $\delta \cdot\left[p\right.$-Methoxystyryl]-4-methoxy- $\alpha$-pyron (Yangonin) $\mathrm{C}_{15} \mathrm{H}_{14} \mathrm{O}_{4} 180$

Necip Güven (Urbana, Illinois), The crystal structures of $2 M_{1}$ phengite and $2 M_{1}$ muscovite . . . . . . . . . . . . . . . . . . 196

Kazuaki Iiishi, Takeshi Tomisaka and Toshio Kats (Yamaguchi) and Yoshiharu Umegaki (Hiroshima), The force field of $\mathrm{K}$ feldspar . . . . . . 213

M. N. Sabesan and K. Venkatesan (Madras), Crystal structure of L-prolylL-phenylalanine-o-methoxy hydrobromide... . . . . . .

T. G. Evans and R. Hine (Cardiff, Wales), The crystal structure of the monopotassium salt of L-aspartic acid dihydrate . . . . . . . . . 243

H. Schulz (Zürich), Influence of heat-treatment on the average structure of $\mathrm{Mg}\left[\mathrm{Al}_{2} \mathrm{Si}_{3} \mathrm{O}_{10}\right]$, a stuffed derivative of the high-quartz structure . .

Joseph R. Smyth (Chicago), Protoenstatite: a crystal-structure refinement at $1100^{\circ} \mathrm{C}$. . . . . . . . . . . . . . . . . . . .

R. F. Giese, Jr., S. Weller and P. Datta (Buffalo, New York), Electrostatic energy calculations of diaspore $(\alpha \mathrm{AlOOH})$, goethite $(\alpha \mathrm{FeOOH})$ and groutite ( $\alpha \mathrm{MnOOH})$. . . . . . . . . . . . . . . . . . . .

Ronald W.T. Wilkins (Queensland), $\mathrm{U}-\mathrm{O}$ bond lengths and force constants in some uranyl minerals

H. Veenvliet and Tiny Migchelsen (Groningen), The crystal structure of $2,3,4,4$-tetrachloro-1-keto-naphthalenedihydride at $-160^{\circ} \mathrm{C}$. . . 291

Gary C. Vezzoli (Fort Monmouth, New Jersey), Proposed structure of highpressure tellurium ......................

B. M. Chadwick, D. W. Jones, J. E. Sarneski, H. J. Wilde and J. Yerkess (Bradford), The coherent neutron scattering amplitude of caesium 308

B. Ribár, B. Matković, M. Šljukić und F. Gabela (Sarajevo und Zagreb), Die Kristallstruktur von $\mathrm{Hg}(\mathrm{OH}) \mathrm{NO}_{3}$. . . . . . . . . . . . . 311

\section{Vorläufige Mitteilungen-Preliminary notices}

M. Ramakrishna Udupa (Göttingen), Crystal data on bis(thiourea)silver(I) thiocyanate. . . . . . . . . . . . . . . . . . . . . 319

C. H.Wong, C. N.Chen, T.H.Lu, K.J.Chao and T.J.Lee (Hsinchu, China), Crystal data for two synergistic complexes, $\mathrm{UO}_{2}$ (TTA) 2 TBP and $\mathrm{UO}_{2}$ (TTA) $)_{2} \mathrm{TOPO}$. . . . . . . . . . . . . . . . . 32 


\section{Heft 5 und 6}

Abgeschlossen Dezember 1971

Roland C. Rouse (Ann Arbor, Michigan), The crystal structure of quenselite 321

W. Prandl (München), Die magnetische Struktur und die Atomparameter des Almandins $\mathrm{Al}_{2} \mathrm{Fe}_{3}\left(\mathrm{SiO}_{4}\right)_{3}$. . . . . . . . . . . . . . . . . . 333

W. Prandl und F. Wagner (München), Die Orientierung des elektrischen Feldgradienten und das innere Magnetfeld beim Almandin. . . . 344

David D. Dexter (New York), The crystal and molecular structure of p-toluene sulfonic acid monohydrate. . . . . . . . . . . . . 350

Masaaki Ohmasa and Werner Nowacki (Bern), The crystal structure of vrbaite $\mathrm{Hg}_{3} \mathrm{Tl}_{4} \mathrm{As}_{8} \mathrm{Sb}_{2} \mathrm{~S}_{20}$. . . . . . . . . . . . . . 360

D. R. Waldbaum (Cambridge, Massachusetts) and R. A. Robie (Silver Spring, Maryland), Calorimetric investigation of $\mathrm{Na}-\mathrm{K}$ mixing and polymorphism in the alkali feldspars. . . . . . . . . . . . . 381

J. D. H. Donnay, Errata in Strukturbericht . . . . . . . . . . . . . . 421

G. Menzer (München), Zu P. Formans historischen Betrachtungen über die Entdeckung der Röntgenstrahl-Interferenzen an Kristallen . . . . 429

12. Diskussionstagung der Sektion für Kristallkunde der Deutschen Mineralogischen Gesellschaft :

R. Brill (Berlin-Dahlem), Carl Hermanns Leben und Werk. Gedächtnisrede anläßlich der 10. Wiederkehr seines Todestages. . . . . . . 438

Referate der Kurzvorträge. . . . . . . . . . . . . . . . . . . . . 448

Autorenregister zu Band 134. . . . . . . . . . . . . . . . . . . . 479

Sachregister zu Band 134 . . . . . . . . . . . . . . . . . . . . . . 482 
.

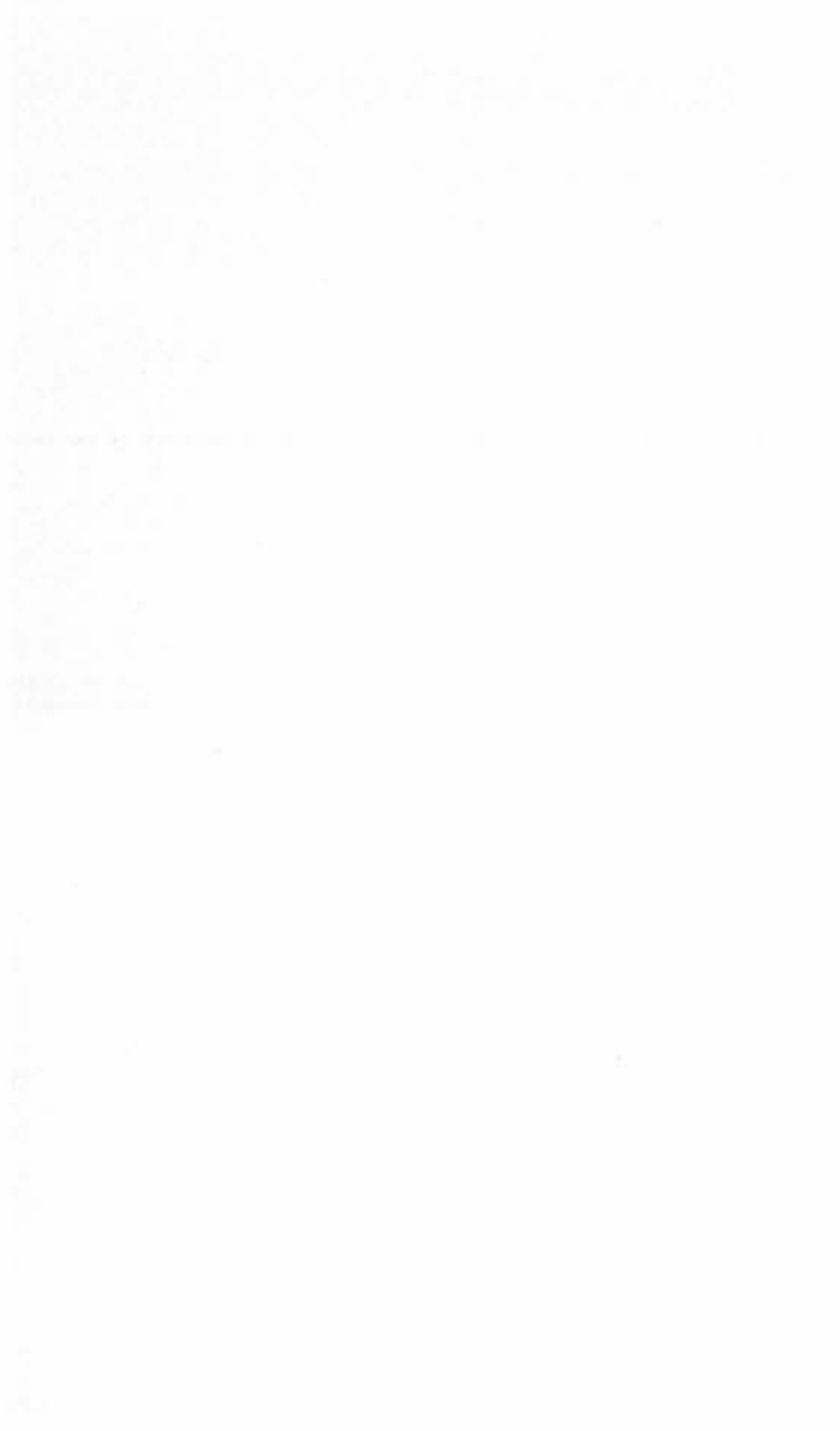

